\title{
Phytoprotection
}

\section{Effet de diverses souches du Trichoderma sur la croissance d'une culture de tomate en serre et leur aptitude à coloniser les racines et le substrat \\ Effect of Trichoderma strains on the growth of tomato plants in greenhouses and their aptitude to colonize roots and substrate}

\author{
Btissam Mouria, Amina Ouazzani-Touhami et Allal Douira
}

Volume 88, numéro 3, 2007

La phytoprotection, 100 ans de découvertes!

Phytoprotection: 100 years of discovery

URI : https://id.erudit.org/iderudit/018955ar

DOI : https://doi.org/10.7202/018955ar

Aller au sommaire du numéro

Éditeur(s)

Société de protection des plantes du Québec (SPPQ)

ISSN

0031-9511 (imprimé)

1710-1603 (numérique)

Découvrir la revue

Citer cet article

Mouria, B., Ouazzani-Touhami, A. \& Douira, A. (2007). Effet de diverses souches du Trichoderma sur la croissance d'une culture de tomate en serre et leur aptitude à coloniser les racines et le substrat. Phytoprotection, 88(3), 103-110. https://doi.org/10.7202/018955ar
Résumé de l'article

Les champignons du genre Trichoderma, connus depuis 1887 pour leurs propriétés antagonistes, ont été utilisés comme agents de lutte biologique contre un large spectre de phytopathogènes. Récemment, certaines études ont démontré l'aptitude des Trichoderma à stimuler la croissance de certaines plantes. Ainsi, l'effet de six souches du Trichoderma sur les paramètres de croissance et de rendement d'une culture de tomate en serre a été étudié. Les résultats montrent que toutes les souches du T. harzianum ont stimulé la croissance de la tomate, notamment les biomasses végétative et racinaire, alors que le $T$. viride n'a pas eu un effet significativement différent par rapport au témoin. L'effet du Trichoderma sur l'incidence des maladies foliaires a aussi été très net pour des souches de $T$. harzianum qui ont protégé à $100 \%$ les feuilles de tomate. La colonisation des racines par les différentes souches du Trichoderma ne diffère pas significativement entre les souches du T. harzianum (86 à $100 \%$ ), alors qu'elle est significativement plus faible pour la souche TV du T. viride. De même, les souches du Trichoderma ont pu se maintenir à un niveau très élevé dans la rhizosphère par rapport aux témoins. 


\title{
Effet de diverses souches du Trichoderma sur la croissance d'une culture de tomate en serre et leur aptitude à coloniser les racines et le substrat
}

\author{
Btissam Mouria', Amina Ouazzani-Touhami et Allal Douira \\ Reçu 2008-02-12; accepté 2008-06-10
}

PHYTOPROTECTION $88: 103-110$

Les champignons du genre Trichoderma, connus depuis 1887 pour leurs propriétés antagonistes, ont été utilisés comme agents de lutte biologique contre un large spectre de phytopathogènes. Récemment, certaines études ont démontré l'aptitude des Trichoderma à stimuler la croissance de certaines plantes. Ainsi, l'effet de six souches du Trichoderma sur les paramètres de croissance et de rendement d'une culture de tomate en serre a été étudié. Les résultats montrent que toutes les souches du T. harzianum ont stimulé la croissance de la tomate, notamment les biomasses végétative et racinaire, alors que le T. viride n'a pas eu un effet significativement différent par rapport au témoin. L'effet du Trichoderma sur l'incidence des maladies foliaires a aussi été très net pour des souches de T. harzianum qui ont protégé à $100 \%$ les feuilles de tomate. La colonisation des racines par les différentes souches du Trichoderma ne diffère pas significativement entre les souches du T. harzianum (86 à $100 \%$ ), alors qu'elle est significativement plus faible pour la souche $\mathrm{TV}_{1}$ du $T$. viride. De même, les souches du Trichoderma ont pu se maintenir à un niveau très élevé dans la rhizosphère par rapport aux témoins.

Mots clés : biomasse racinaire, biomasse végétative, croissance, stimulation, tomate, Trichoderma.

[Effect of Trichoderma strains on the growth of tomato plants in greenhouses and their aptitude to colonize roots and substrate]

Fungi in the genus Trichoderma, known since 1887 for their antagonistic properties, have been used as a biological control organism against several plant pathogens. Recently, studies showed the aptitude of Trichoderma to stimulate plant growth. Thus, the effect of six strains of Trichoderma on greenhouse tomato growth and yield was studied. The results show that all T. harzianum strains stimulated tomato growth, in particular the shoot and root weights, whereas $T$. viride did not have a significant effect. The effect of Trichoderma on leaf disease incidence was very clear since tomato leaves were completely protected $(100 \%)$ by some T. harzianum strains. Root colonization by the various strains of Trichoderma did not differ significantly between the T. harzianum strains (86 to $100 \%$ ), whereas it was significantly lower for the $\mathrm{TV}_{1}$ strain of $T$. viride. Also, Trichoderma strains maintained a high concentration level in the rhizosphere compared with the controls.

Keywords: Growth, root weight, shoot weight, stimulation, tomato, Trichoderma.

\section{INTRODUCTION}

Les propriétés antagonistes du Trichoderma ont été mentionnées pour la première fois par Vuillemin en 1887 (cité dans Lamy Krafft et Roquebert 1981). Ce n'est toutefois qu'en 1971 que Dennis et Webster ont pu élucider les différents mécanismes d'action de ce champignon antagoniste qui incluent principalement le mycoparasitisme, I'antibiose et la compétition pour les nutriments et l'espace (Dennis et Webster 1971a, b, c).

Depuis, les recherches portant sur ce champignon se sont multipliées (Lamy Krafft et Roquebert 1981). Ainsi, les champignons du genre Trichoderma ont été utilisés comme agents de lutte biologique contre un large spectre de pathogènes aussi bien telluriques
(Camporota 1985; Davet 1986; Ouazzani-Touhami et al. 1994) que foliaires (Hmouni et al. 1999; Mouria et al. 1997a, b).

Plus récemment, les travaux de Baker (1988) et de Lynch et al. (1991a) ont montré que certaines souches du Trichoderma semblaient exercer une action stimulatrice sur la croissance de certaines plantes. En effet, Lynch et al. (1991a, b) ont étudié l'effet du Trichoderma sur la croissance de la laitue et son aptitude à lutter contre le Rhizoctonia solani Kühn et le Pythium ultimum Trow, des agents de la fonte des semis. Ils ont aussi démontré l'effet de certaines souches du Trichoderma sur la croissance de la laitue et la germination des graines en l'absence de tout agent pathogène. Cependant, cette découverte n'a pas donné lieu à d'autres recherches.

1. Laboratoire de Botanique et de Protection des Plantes, Faculté des Sciences, Université Ibn Tofaïl, B.P. 133, Kénitra, Maroc; courriel : labobotanique@gmail.com 
Au Maroc, aucune étude n'a porté sur l'utilisation du Trichoderma comme stimulateur de croissance des plantes. L'objectif de la présente étude était donc de déterminer l'effet de diverses souches du Trichoderma harzianum Rifai et du T. viride Pers. sur la croissance et le rendement d'une culture de tomate en serre.

\section{MATÉRIEL ET MÉTHODES}

\section{Cultivar de tomate}

Des semences de tomate appartenant à la variété Campbell 33 ont été désinfectées à l'hypochlorite de sodium dilué à $1 \%$ pendant 10 min, puis rincées abondamment sous un courant d'eau. Après séchage, les graines ont été mises à prégermer dans des plateaux alvéolés à 77 trous remplis de tourbe noire, dont la composition est présentée au tableau 1, à raison d'une graine par alvéole. Les plateaux ont été couverts d'un film plastique de $20 \mu \mathrm{m}$ d'épaisseur pendant $2 \mathrm{j}$ pour assurer une bonne germination.

Le repiquage des plantules a été effectué lorsque celles-ci ont atteint le stade de deux vraies feuilles bien étalées (Woo et al. 1996), soit 18 j après semis, dans des pots de $17 \mathrm{~cm} \times 14 \mathrm{~cm}(4 \mathrm{~L})$ en polyéthylène perforés à la base.

\section{Agents de lutte biologique}

Les souches du Trichoderma utilisées dans cette étude ont diverses origines. La souche Tctom a été isolée à partir de feuilles de plants de tomate appartenant à la variété Campbell 33 cultivés sur un substrat constitué uniquement de sol; Tgraine a été isolée à partir de graines du Ricinus traitées par le thiabendazole et mises à prégermer dans des chambres humides; Tcomp a été isolée à partir d'un compost de déchets urbains en utilisant la méthode de suspensions-dilutions sur milieu sélectif $S$ de Messiaen et Lafon (1965) à faible teneur en sucre réduit et sans extrait de Malt, additionné d'un fongicide, la vinchlozoline $\left(2,5 \mathrm{mg} \mathrm{L}^{-1}\right)$, peu inhibiteur pour le Trichoderma (Davet 1979) et de $1 \mathrm{~g} \mathrm{~L}^{-1}$ de $\mathrm{CaCl}$ qui favorise la sporulation (Gindrat 1977). La prolifération des bactéries a été évitée en ajoutant du chloramphénicol dans le milieu à raison de $100 \mathrm{mg} \mathrm{L}^{-1}$.

Les souches $\mathrm{TH}_{1}$ et $\mathrm{TH}_{2}$ du Trichoderma harzianum et $\mathrm{TV}_{1}$ du $T$. viride, appartenant à la mycothèque du LBPP (Laboratoire de Botanique et de Protection des Plantes) et connues pour leur activité antagoniste très élevée (Hmouni et al. 1999; Mouria et al. 1997a, b; Ouazzani-Touhami et al. 1997a, b), ont été utilisées à titre comparatif.

L'identification des souches Tctom, Tcomp et Tgraine a été effectuée en se basant sur leurs caractères morphologiques; elles appartiennent à l'espèce Trichoderma harzianum. La vérification de l'identification a été réalisée au Muséum national d'Histoire naturelle de Paris. Toutes les souches ont été conservées sur papier filtre à $-15^{\circ} \mathrm{C}$.

\section{Procédé d'inoculation}

Les six souches du Trichoderma ont été cultivées sur milieu PDA et incubées à $28^{\circ} \mathrm{C}$ pendant 5 j à l'obscurité et $5 \mathrm{j}$ de lumière afin de favoriser leur sporulation.
Des suspensions sporales ont été préparées en raclant la surface de ces cultures immergées avec de l'eau distillée stérile. Les filtrats ont été obtenus en passant les suspensions à travers quatre couches de gaze et les concentrations ont été ajustées à $10^{7}$ spores $\mathrm{mL}^{-1}$ à l'aide d'un hématimètre.

L'inoculation a été réalisée par trempage des racines des plantules de tomate enrobées de leur substrat de germination dans les suspensions conidiennes pendant $30 \mathrm{~min}$. Les plantules ont par la suite été repiquées dans des pots contenant du sol de la forêt Mamora. C'est un sol très sableux $(91,1 \%$ de sable) à structure meuble, de $\mathrm{pH}$ légèrement basique (Zidane 2004) présentant une faible capacité d'échange cationique ( $\left.7 \mathrm{mEq} 100 \mathrm{~g} \mathrm{~g}^{-1}\right)$ et une très faible salinité. Les pots ont ensuite été placés dans une serre de culture pendant 5 sem à une température de 18 à $25^{\circ} \mathrm{C}$ et une photopériode de $12 \mathrm{~h} \mathrm{~L}: 12 \mathrm{~h} \mathrm{O}$.

Le protocole expérimental a été conçu en blocs aléatoires avec cinq répétitions pour chaque souche et un plant par pot. Les plants témoins ont subi un trempage des racines dans l'eau stérile au lieu de la suspension sporale. L'arrosage était effectué tous les 2 j à l'aide d'eau du robinet. Cette fréquence d'arrosage est suffisante pour une culture de tomate en pots parce que le drainage est faible, notamment quand la température est basse et que l'évaporation est faible.

\section{Notation des résultats}

A la fin des essais, les pots ont été ramenés de la serre et les pousses ont été coupées au niveau du collet. La hauteur des pousses a été mesurée du collet à l'insertion de la plus jeune feuille. Le nombre de feuilles, de fleurs et de fruits par plant a été compté et le pourcentage de feuilles présentant des lésions ou des nécroses a été calculé selon l'équation suivante :

$$
\% \mathrm{Fa}=\frac{\mathrm{N}_{\mathrm{Fa}}}{\mathrm{N}_{\mathrm{TF}}} \times 100
$$

où : \% $\mathrm{Fa}=$ pourcentage de feuilles attaquées; $\mathrm{N}_{\mathrm{Fa}}=$ nombre de feuilles attaquées; et NTF = nombre total de feuilles.

Les racines ont été séparées du sol par un léger mouvement et lavées sous un courant d'eau afin d'éliminer les particules de sol adhérentes. Les racines de trois plants ont été utilisées pour déterminer le poids frais et sec, alors que celles des deux autres ont été utilisées pour la mise en évidence de la colonisation par le Trichoderma.

La biomasse végétative a été mesurée à l'aide d'une balance de précision (Bosch-Wägesysteme GmbH D-7241, Jungingen, Germany) le même jour alors que la biomasse racinaire a été mesurée après égouttage sur papier absorbant pendant une nuit dans les conditions ambiantes du laboratoire. Les poids secs des pousses et des racines ont été déterminés après séchage à $43{ }^{\circ} \mathrm{C}$ pendant $4 \mathrm{j}$ (McGovern et al. 1992). 


\section{Colonisation des racines}

\section{et maintien dans le substrat}

Afin de mettre en évidence la colonisation des souches du Trichoderma dans le système racinaire des plants de tomate, les racines de deux plants pour chaque souche ont été lavées, désinfectées superficiellement à l'alcool $90^{\circ}$ pendant $1 \mathrm{~min}$, rincées abondamment à l'eau distillée stérile, séchées, découpées en petits morceaux et déposées dans des boîtes de Pétri contenant de l'eau gélosée.

Le pourcentage de colonisation des racines a été calculé à partir du nombre de fragments de racines entourés par un mycélium du Trichoderma selon la relation ci-dessous. Seize fragments de racines ont été déposés par boîte de Pétri et chaque plante a fait l'objet de trois répétitions.

$$
\% \text { Co }=\frac{\mathrm{N}_{\mathrm{fc}}}{\mathrm{N}_{\mathrm{Tf}}} \times 100
$$

où : $\%$ Co $=$ pourcentage de colonisation; $\mathrm{N}_{\mathrm{fc}}=$ nombre de fragments colonisés; et $\mathrm{N}_{\mathrm{Tf}}=$ nombre total de fragments.

Par ailleurs, l'estimation de la population du Trichoderma dans le substrat de culture a été menée sur deux types d'échantillons à raison de cinq répétitions pour chaque échantillon :

- sol de la rhizosphère en contact direct avec les racines; et

- sol à distance des racines prélevé à $2 \mathrm{~cm}$ de la bordure des pots et à $5 \mathrm{~cm}$ de profondeur.

En utilisant la technique des suspensions-dilutions, $0,1 \mathrm{~mL}$ de chaque dilution a été étalé sur milieu $\mathrm{S}$ de Messiaen et Lafon (1965) modifié. Le dénombrement des colonies a été effectué après $64 \mathrm{~h}$ d'incubation à $25^{\circ} \mathrm{C}$. Le nombre de propagules par $\mathrm{g}$ de sol a été calculé selon la formule suivante :

$$
\text { Nombre UFC g }{ }^{-1} \text { de sol }=\frac{\mathrm{NC}}{0,1 \mathrm{~mL}} \times \mathrm{Fd}
$$

où : UFC = unités formant des colonies; NC = nombre de colonies; et $\mathrm{Fd}=$ facteur de dilution.

\section{Analyses statistiques}

Le traitement des données a consisté en une analyse de variance et un test PPDS ou de comparaison planifiée, basé sur la théorie de stimulation de la croissance des plants, au seuil de $5 \%$ à l'aide du logiciel STATISTICA. Les analyses statistiques ont porté sur les résultats des trois plants pour la partie stimulation de la croissance, sur les trois répétitions de chacun des deux autres plants pour la colonisation des racines et sur les cinq répétitions dans le cas du maintien du Trichoderma dans le sol et la rhizosphère.

\section{RÉSULTATS}

\section{Effet du Trichoderma sur les paramètres de rendement}

L'effet de l'inoculation des racines des plants de tomate par les suspensions sporales des différentes souches du Trichoderma sur les paramètres de rendement est présenté dans le tableau 1.

L'analyse de la variance au seuil de $5 \%$ a montré que la souche $\mathrm{TH}_{1}$ du $T$. harzainum a induit la meilleure réponse de stimulation des paramètres de croissance des plants de tomate. En effet, les plantes inoculées par cette souche ont montré une meilleure croissance axiale (hauteur des pousses) et une meilleure genèse des feuilles et des fleurs (Tableau 1 et Fig. 1A). De même, les poids frais et secs des pousses, et surtout des racines de ces plantes, ont surpassé ceux des autres lots (Tableau 1).

\begin{tabular}{|c|c|c|c|c|c|c|c|c|}
\hline \multirow[b]{2}{*}{ Traitement } & \multicolumn{8}{|c|}{ Paramètres } \\
\hline & $\begin{array}{l}\text { Taille } \\
\text { (cm) }\end{array}$ & $\begin{array}{l}\text { Feuilles } \\
\text { (nb/plant) }\end{array}$ & $\begin{array}{c}\text { Feuilles } \\
\text { attaquées } \\
\\
(\%)\end{array}$ & $\begin{array}{l}\text { Poids } \\
\text { frais des } \\
\text { pousses } \\
\text { (g) }\end{array}$ & $\begin{array}{c}\text { Poids sec } \\
\text { des } \\
\text { pousses } \\
\text { (g) }\end{array}$ & $\begin{array}{l}\text { Poids } \\
\text { frais des } \\
\text { racines } \\
(\mathrm{g})\end{array}$ & $\begin{array}{c}\text { Poids sec } \\
\text { des } \\
\text { racines } \\
(\mathrm{g})\end{array}$ & $\begin{array}{c}\text { Fleurs } \\
\text { (nb/plant) }\end{array}$ \\
\hline Témoin & $38,83 d^{2}$ & $10,33 \mathrm{~b}$ & 22,42 a & $15,30 \mathrm{~d}$ & $2,46 d$ & 5,53 e & $0,56 \mathrm{~d}$ & $4,33 \mathrm{c}$ \\
\hline $\mathrm{TH}_{1}$ & 56,66 a & $13,66 \mathrm{a}$ & $0,00 \mathrm{~b}$ & $42,90 \mathrm{a}$ & 7,53 a & $16,80 \mathrm{a}$ & $1,53 \mathrm{a}$ & $14,33 \mathrm{a}$ \\
\hline $\mathrm{TH}_{2}$ & $52,00 \mathrm{bc}$ & $12,66 \mathrm{ab}$ & $0,00 \mathrm{~b}$ & $32,20 \mathrm{c}$ & $5,56 \mathrm{~b}$ & $13,73 \mathrm{~b}$ & $1,30 \mathrm{ab}$ & $11,66 \mathrm{ab}$ \\
\hline Tcomp & $54,16 \mathrm{ab}$ & $12,33 \mathrm{ab}$ & $0,00 \mathrm{~b}$ & $38,23 \mathrm{~b}$ & $8,03 \mathrm{a}$ & $10,83 \mathrm{c}$ & $1,07 \mathrm{bc}$ & $7,66 \mathrm{bc}$ \\
\hline Tctom & $50,66 \mathrm{c}$ & $11,00 \mathrm{~b}$ & $0,00 \mathrm{~b}$ & $30,46 \mathrm{c}$ & $5,13 \mathrm{bc}$ & 7,96 de & $0,87 \mathrm{c}$ & $8,00 \mathrm{bc}$ \\
\hline Tgraine & $50,33 \mathrm{c}$ & $11,33 a b$ & $5,80 \mathrm{~b}$ & $30,50 \mathrm{c}$ & $4,43 \mathrm{c}$ & $10,06 \mathrm{~cd}$ & $1,05 \mathrm{bc}$ & $7,66 \mathrm{bc}$ \\
\hline $\mathrm{TV}_{1}$ & $41,00 \mathrm{~d}$ & $11,00 \mathrm{~b}$ & $9,09 \mathrm{~b}$ & $18,80 \mathrm{~d}$ & $2,53 \mathrm{~d}$ & $5,40 \mathrm{e}$ & $0,28 d$ & $6,33 \mathrm{c}$ \\
\hline
\end{tabular}

Tableau 1. Variation des paramètres de rendement des plants de tomate inoculés par différentes souches de Trichoderma

${ }^{1}$ Feuilles présentant des lésions ou des nécroses.

${ }^{2}$ Dans chaque colonne, les valeurs suivies de la même lettre ne diffèrent pas significativement au seuil de $5 \%$ selon le test de PPDS ou de comparaison planifiée. 
A

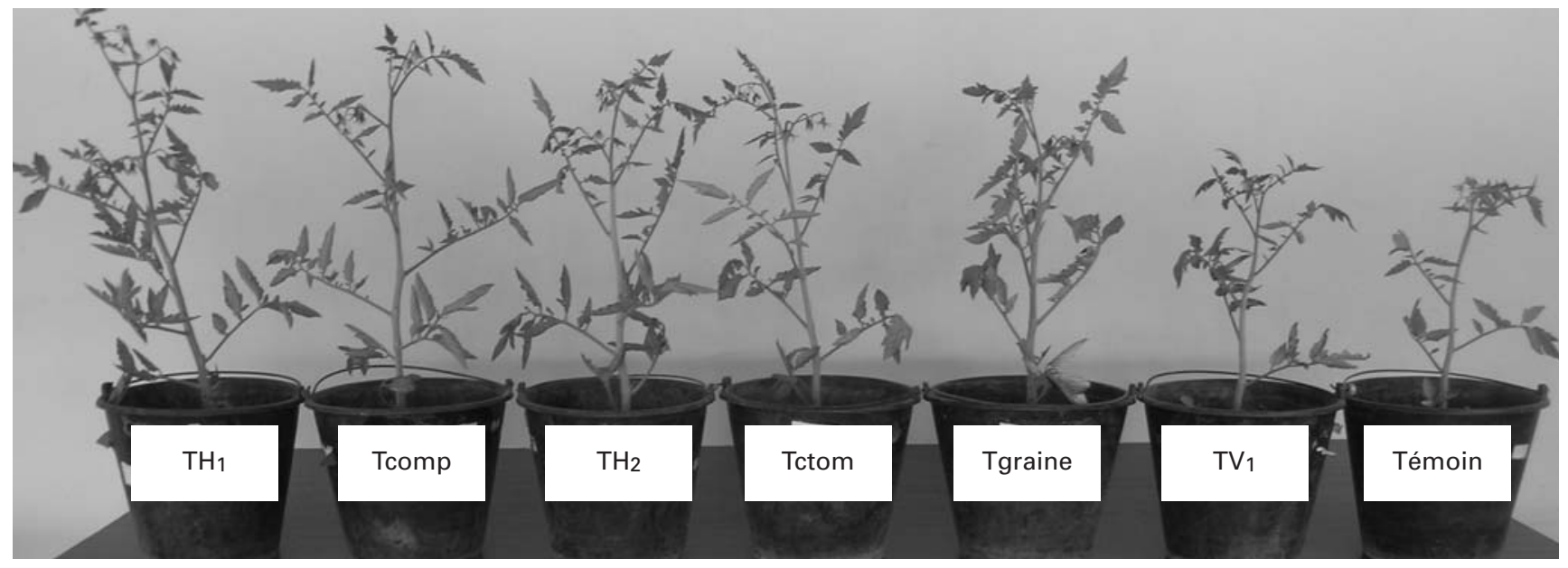

B

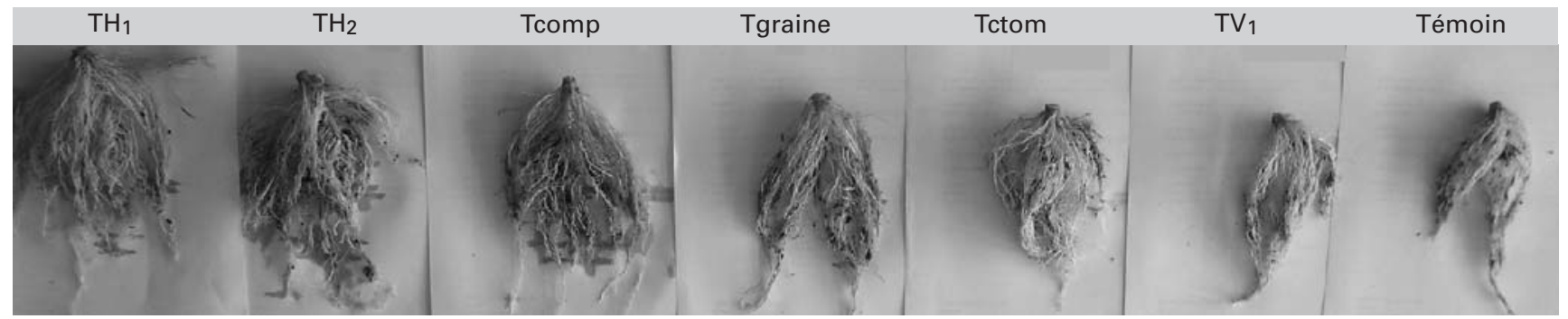

C

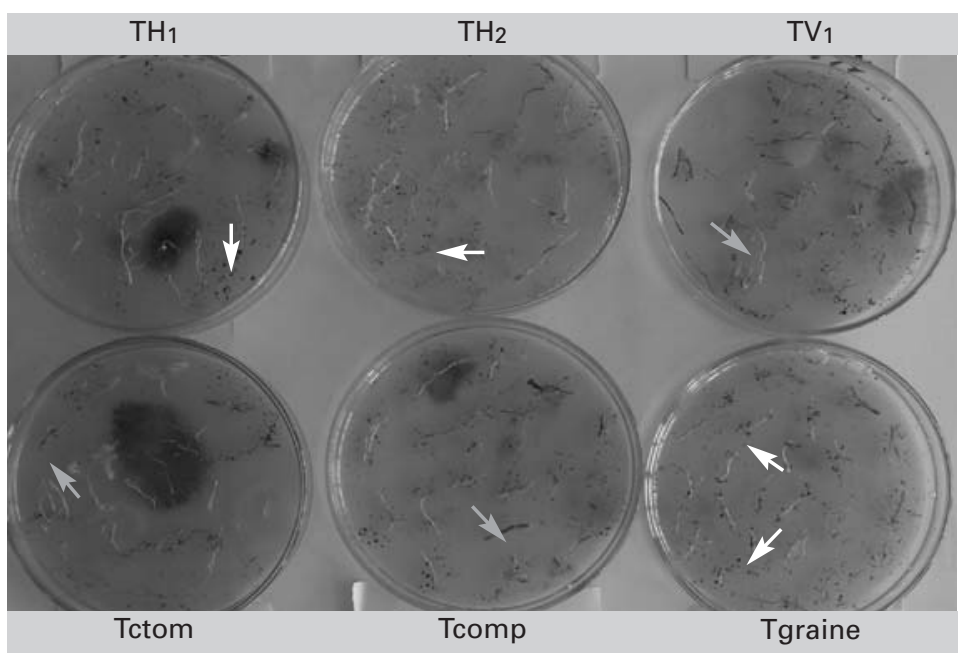

Figure 1. Effet de l'inoculation des racines des plants de tomate par différentes souches du Trichoderma sur (A) la croissance et le développement des plants de tomate; (B) le développement du système racinaire; (C) la colonisation des racines (les flèches blanches indiquent les propagules du Trichoderma et les flèches grises indiquent les fragments de paille). 


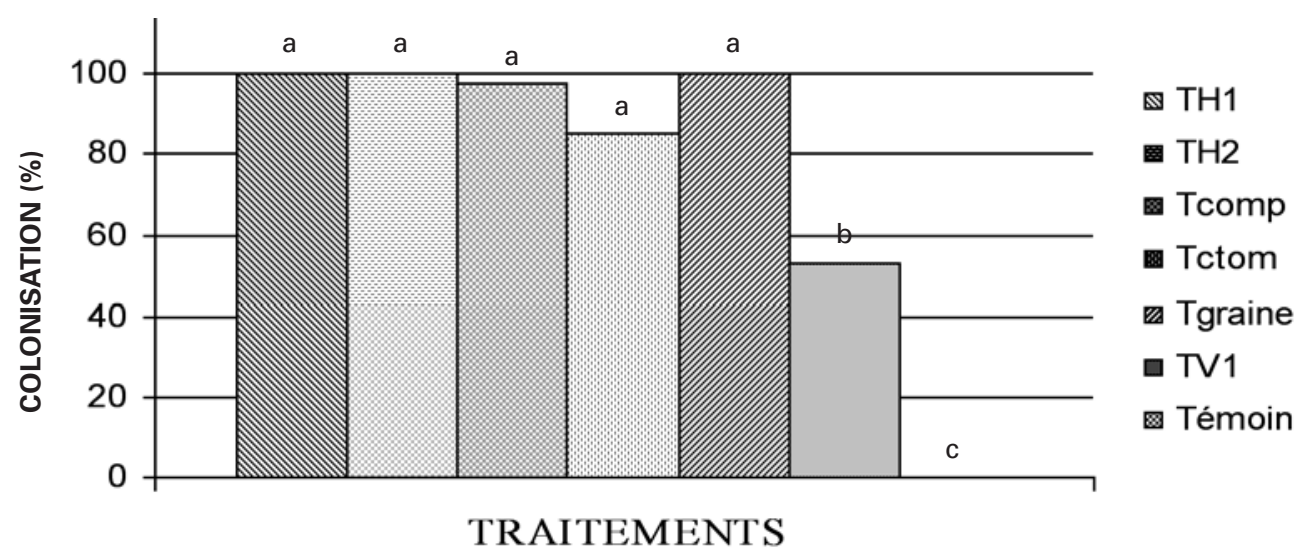

Figure 2. Colonisation des racines des plants de tomate par différentes souches du Trichoderma.

Les souches $\mathrm{TH}_{2}$, Tcomp, Tctom et Tgraine ont induit une réponse nettement supérieure à celle des témoins non inoculés, en particulier la souche Tcomp qui a engendré une croissance axiale, un nombre de feuilles et une biomasse végétative proches de ceux engendrés par la souche $\mathrm{TH}_{1}$. La souche $\mathrm{TH}_{2}$ a plutôt rivalisé avec la souche $\mathrm{TH}_{1}$ en nombre de feuilles, en biomasse racinaire et en nombre de fleurs (Tableau 1 et Fig. 1B).

Une seule plante, inoculée par la souche Tctom, a produit un fruit à la fin de l'essai, ce qui n'a pas eu d'effet significatif au seuil de $5 \%$ par rapport au témoin.

La souche $\mathrm{TV}_{1}$ du $T$. viride est la seule parmi les six souches testées qui n'a pas eu d'effet positif sur les plantes inoculées. En effet, pour cette souche, les paramètres de croissance des plants de tomate ont souvent été similaires à ceux des témoins.

D'un autre côté, les souches $\mathrm{TH}_{1}, \mathrm{TH}_{2}$, Tcomp et Tctom ont permis une protection totale des feuilles de tomate qui n'ont présenté aucun symptôme de détérioration, alors que les plantes inoculées par les souches Tgraine et $\mathrm{TV}_{1}$ ont présenté des lésions $(5,80$ et $9,09 \%$ des feuilles) engendrées surtout par des champignons du genre Alternaria. Cependant, l'incidence de ces symptômes demeure largement inférieure, au seuil de $5 \%$, à celle des plantes témoins dont $22,42 \%$ montraient des lésions et des nécroses. De même, le diamètre des lésions sur les plantes témoins était nettement plus élevé que chez les plantes traitées par ces deux souches du Trichoderma (données non présentées).

\section{Colonisation des racines par le Trichoderma}

Les pourcentages de colonisation des racines des plants de tomate par les différentes souches du Trichoderma sont illustrés dans la figure 2.

À l'exception de la souche $T V_{1}$, il n'y a pas de différence significative entre la capacité des Trichoderma utilisés à coloniser les racines des plants de tomate. Cependant, il existe une différence entre l'aptitude de ces souches à empêcher les champignons du sol de pénétrer dans les racines (Fig. 1C). La plus faible croissance de ces champignons a été enregistrée en présence des souches Tgraine, $\mathrm{TH}_{2}$ et
Tcomp. En effet, le repiquage sur milieu gélosé des racines des plantes inoculées par la souche Tgraine n'a révélé la présence d'aucune autre espèce de champignons.

L'identification des espèces isolées à partir des racines a montré la dominance des champignons suivants : Alternaria alternata (Fr. keissl.), Aspergillus fumigatus Fresen., Helminthosporium spiciferum L., Fusarium sp. et Ulocladium atrum Preuss.

\section{Maintien du Trichoderma dans le substrat de culture}

La figure 3 illustre les niveaux des populations des souches du Trichoderma à la fin de l'essai dans le substrat de culture des plants de tomate, au niveau de la rhizosphère (Fig. $3 \mathrm{~A}$ ) et à distance des racines (Fig. 3B).

Ainsi, les différentes souches du Trichoderma ont pu se maintenir au niveau de la rhizosphère à des concentrations plus élevées que celles décelées à distance des racines. Ces dernières demeurent toutefois nettement supérieures à celles rencontrées au niveau du sol et de la rhizosphère des lots témoins, où l'apparition des colonies du Trichoderma était occasionnelle.

\section{DISCUSSION}

Davet et al. (1981) ont montré que lors de l'inoculation du sol par le Trichoderma, il est nécessaire d'introduire l'inoculum avec un support qui lui fournit une base nutritive suffisante pour échapper, au moins temporairement, à la compétition des autres microorganismes telluriques. Ainsi, la tourbe, adhérant aux racines des plantules, est utilisée comme support physique afin de permettre à l'inoculum du Trichoderma de se multiplier au cours des premiers jours loin de l'influence des populations microbiologiques du sol.

Les résultats montrent que presque toutes les souches du Trichoderma sont parvenues à stimuler les paramètres de croissance des plants de tomate à des degrés variables. Cette stimulation s'est traduite essentiellement par une meilleure croissance axiale 


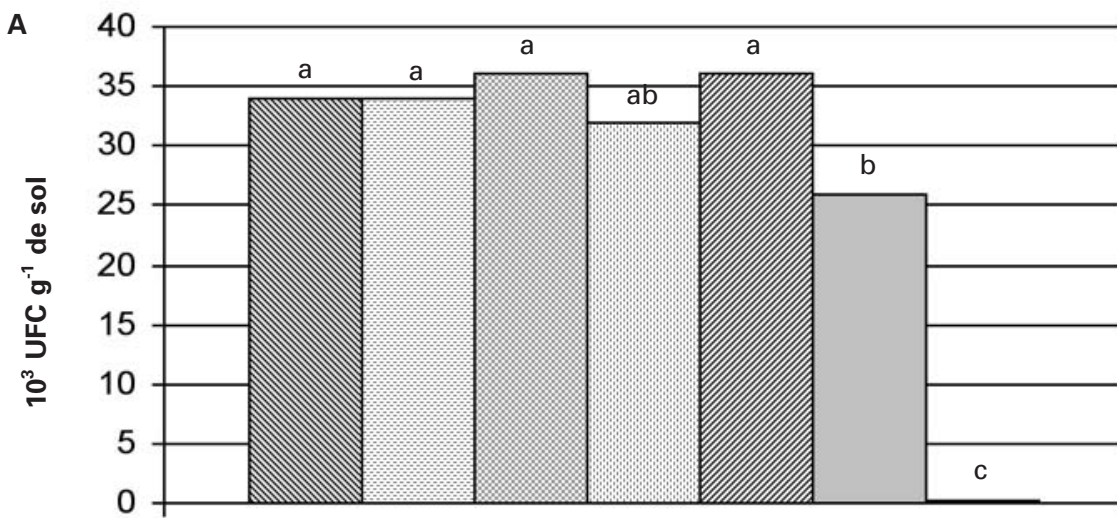

TRAITEMENTS

B

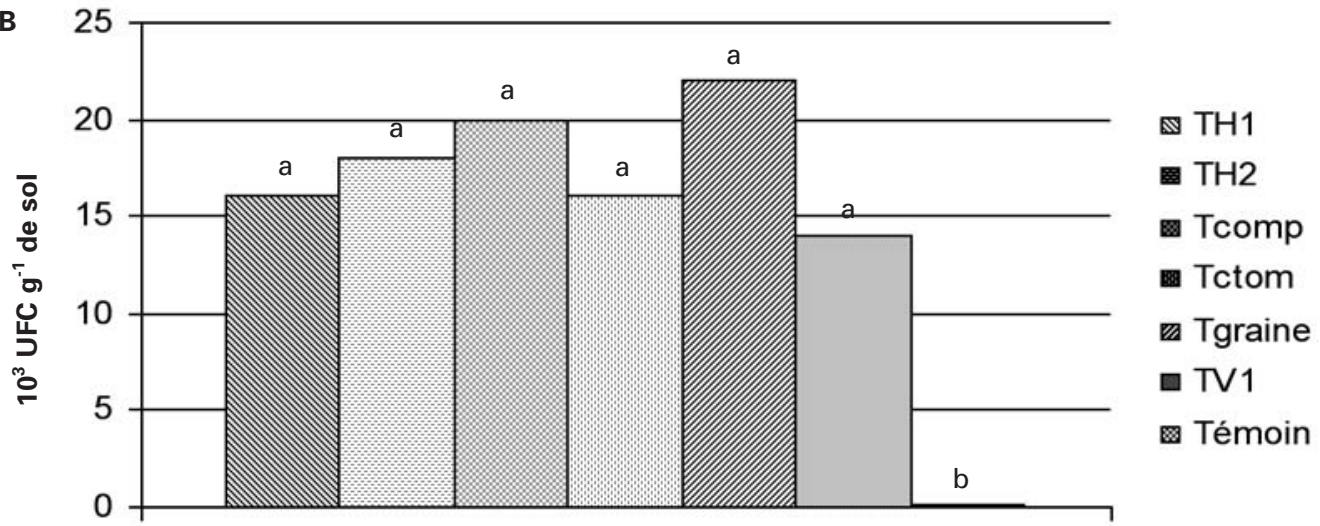

TRAITEMENTS $\mathbb{\mathrm { TH }} 1$

$\mathrm{TH} 2$

Tcomp

Tctom

Tgraine

TV1

Témoin des racines, à la fin de l'essai.

et une biomasse plus importante. La stimulation de la biomasse a été observée non seulement dans les parties aériennes, mais aussi dans les parties racinaires qui ont augmenté de 270 à $325 \%$ par rapport au témoin. Cette augmentation a été notée aussi bien pour les poids frais que pour les poids secs, ce qui montre qu'il s'agit d'un accroissement général du métabolisme et non seulement d'une absorption excessive d'eau.

Ceci est en accord avec les travaux de Windham et al. (1986) qui ont démontré que l'addition du T. harzianum et du T. koningii Oudermans à un sol préalablement autoclavé augmentait le poids sec des racines ainsi que de la partie aérienne de la tomate et du tabac de 213 à $291 \%$ par rapport au témoin non traité. De même, les travaux de Baker et al. (1984) ont montré que le poids sec de plantes de radis avait augmenté de plus de $274 \%$ après traitement par la souche T-95 du T. harzianum.

En outre, Gravel et al. (2005) ont démontré que l'inoculation de la tomate avec une souche du T. atroviride
P. Karst permettait d'augmenter significativement la surface radiculaire et le poids des plantules de tomate in vitro ainsi que le rendement commercialisable en serre.

Mieux encore, Hibar et al. (2005) ont comparé l'état des plants de tomate inoculés par une souche pathogène du F. oxysporum Schlechtend.:Fr. et par $T$. harzianum avec des plants témoins sains non inoculés et non traités. Ils ont constaté que les plants traités par le pathogène et l'antagoniste présentaient un développement végétatif et racinaire plus important que les plants témoins.

Les souches du T. harzianum ont amélioré les paramètres de croissance des plantes, alors que le T. viride a induit une réponse similaire à celle des témoins. En effet, la majorité des travaux qui ont démontré l'effet stimulateur de croissance ont porté sur l'espèce T. harzianum. Ainsi, Besnard et Davet (1993) ont sélectionné, à partir de 100 souches de Trichoderma, deux souches du T. harzianum ayant exercé un effet à la fois stimulant sur le pourcentage 
de germination, la matière sèche et le nombre moyen de fleurs des plants de tomate et de concombre, et inhibant sur le Pythium.

Bien que les mécanismes de stimulation de la croissance des plantes par le Trichoderma ne soient pas encore bien élucidés, ceux-ci sont souvent attribués à leur aptitude à combattre les maladies des plantes. Dans ce sens, Hibar et al. (2005) ont expliqué la stimulation du développement d'une culture de melon à la suite de l'application du T. harzianum (Yedidia et al. 1999) par une activation du système de défense de la plante, une augmentation de l'activité chitinase et peroxidase et un accroissement de l'activité enzymatique dans les feuilles induisant une résistance systémique chez ces plants. Par contre, Ousley et al. (1993) attribuent ce phénomène à l'inhibition des pathogènes mineurs, induisant ainsi une forte croissance et un meilleur approvisionnement des nutriments.

Dans cette étude, toutes les souches du Trichoderma inoculées, en particulier T. harzianum, ont réduit le pourcentage de lésions sur les feuilles de tomate par rapport aux témoins, ce qui confirme que cette réduction est en partie responsable de la stimulation de croissance chez ces plantes.

Cependant, d'autres travaux ont mis en évidence l'aptitude des espèces du Trichoderma à stimuler la croissance des plantes indépendamment de tout agent pathogène (Chang et al. 1986; Kleifeld et Chet 1992; MacKenzie et al. 1995; Paulitz et al. 1986; Windham et al. 1986), ce qui est d'ailleurs confirmé dans cette étude. Ceci montre que ces souches influencent le métabolisme et l'activité enzymatique des plantes et non pas seulement les systèmes de défense.

Windham et al. (1986) ont rapporté que les souches de Trichoderma étudiées produisaient des régulateurs de croissance qui ont amélioré le taux de germination et les poids secs des pousses. Baker (1988) a donc conclu que les réponses de stimulation des plantes par le Trichoderma sont dues aussi bien à son aptitude à lutter contre les pathogènes mineurs qu'à la production de régulateurs de croissance.

Une des caractéristiques essentielles chez un agent de lutte biologique est son aptitude à survivre dans un milieu différent de son milieu d'origine et à coloniser les racines des plantes afin de les protéger contre les pathogènes (Nemec et al. 1996). Dans cette étude, toutes les souches du Trichoderma testées ont pu coloniser les racines des plantes inoculées. Cependant, leur aptitude à la compétition avec les autres microorganismes du sol a différé d'une souche à une autre. Les souches Tgraine, Tcomp et $\mathrm{TH}_{2}$ ont semblé plus compétitives vis-à-vis des microorganismes du sol que les autres souches de Trichoderma.

Ces résultats confirment les travaux de Kleifeld et Chet (1992) qui ont rapporté que la stimulation de croissance des plantes par le $T$. harzianum serait due à l'augmentation du transfert des nutriments à partir du sol jusqu'aux racines grâce à la colonisation de celles-ci par le Trichoderma.

Ousley et al. (1994) précisent que le T. harzianum améliore l'acheminement des nutriments du compost jusqu'aux racines d'une manière similaire aux effets des mycorhizes. Ces auteurs ont d'ailleurs supposé que la réponse de stimulation des plantes est due à la production de métabolites thermostables qui stimulent directement la croissance des plantes ou à la capacité du T. harzianum à inactiver les matières toxiques du sol qui inhibent la croissance des plantes.

En 2006, Harman a cité des recherches récentes montrant que les effets du Trichoderma sur les plantes incluent l'induction d'une résistance systémique ou localisée. Ces champignons colonisent l'épiderme des racines et les couches corticales externes et libèrent des molécules bioactives. En conséquence, en plus de l'induction de la résistance chez les plantes, la croissance des plantes et l'approvisionnement en nutriments sont améliorés.

Toutefois, seule l'identification de composés responsables de la stimulation de croissance et la démonstration de leur effet sur des plantes cultivées en serre devraient permettre de conclure sur le mécanisme mis en jeu (Besnard et Davet 1993).

D'autre part, Kleifield et Chet (1992) ont rapporté que la stimulation des plantes par le Trichoderma dépend de sa capacité à survivre et à se maintenir dans la rhizosphère. Pour cela, l'estimation des populations du Trichoderma dans le substrat de culture à la fin des essais a montré leur capacité à se maintenir à un niveau élevé dans la rhizosphère; cependant, ce niveau varie d'une souche à l'autre.

Le classement des souches selon la population estimée au niveau de la rhizosphère, mais surtout à distance des racines où la compétitivité est plus prononcée, rappelle celui obtenu selon leur aptitude compétitive. Ceci est en accord avec les travaux d'Ozbay et Newman (2004) qui ont observé que les souches utilisées ont maintenu leurs populations à des niveaux élevés 4 sem après inoculation. De même, Besnard et Davet (1993) ont constaté que les populations du Trichoderma se sont maintenues à la fin de leur expérience au niveau de l'inoculum introduit.

Nous concluons donc de cette étude que les souches du T. harzianum, en plus de leur effet protecteur contre les pathogènes, exercent un effet stimulateur sur la croissance des plants de tomate. L'éventuel mécanisme portant sur la sécrétion de métabolites ou de régulateurs de croissance nécessite d'autres recherches pratiques pour pouvoir le confirmer.

L'inoculation des racines de plantules de tomate entourées de leur substrat de germination constitue donc un moyen efficace, commode et peu coûteux d'obtenir des plantes vigoureuses, protégées contre les phytopathogènes et avec un meilleur rendement.

Par ailleurs, l'application du T. harzianum en agriculture est une approche prometteuse qui permet de réduire l'utilisation des fongicides et des régulateurs de croissance tout en minimisant le coût de production et les impacts négatifs sur l'environnement. 


\section{RÉFÉRENCES}

Baker, R. 1988. Trichoderma spp. as plant-growth stimulants, CRC Crit. Rev. Biotechnol. 7 (2) : 97-106.

Baker, R., Y. Elad et I. Chet. 1984. The controlled experiment in the scientific method with special emphasis on biological control. Phytopathology 74 : 1019-1021.

Besnard, O. et P. Davet. 1993. Mise en évidence de souches de Trichoderma spp. à la fois antagonistes de Pythium ultimum et stimulatrices de la croissance des plantes. Agronomie $13: 413-421$.

Camporota, P. 1985. Antagonisme in vitro de Trichoderma spp. vis-à-vis de Rhizoctonia solani Kühn. Agronomie 5 (7) : 613-620.

Chang, Y.C., Y.C. Chang, R. Baker, O. Kleifeld et I. Chet. 1986. Increased growth of plants in the presence of the biological control agent Trichoderma harzianum. Plant Dis. $70(2): 145-148$.

Davet, P. 1979. Technique pour l'analyse des populations de Trichoderma et de Gliocladium virens dans le sol. Ann. Phytopathol. 11 (4) : 529-533.

Davet, P. 1986. Activité parasitaire des Trichoderma vis-à-vis des champignons à sclérotes; corrélation avec l'aptitude à la compétition dans un sol non stérile. Agronomie 6 (9) : 863-867.

Davet, P., M. Artigues et C. Martin. 1981. Productions en conditions non aseptiques d'inoculum de Trichoderma harzianum Rifai pour des essais de lutte biologique. Agronomie 1 (10): 933-936.

Dennis, C. et J. Webster. 1971a. Antagonistic properties of species groups of Trichoderma. I. Production of non volatile antibiotics. Trans. Br. Mycol. Soc. 57 : 25-39.

Dennis, C. et J. Webster. 1971b. Antagonistic properties of species groups of Trichoderma. II. Production of volatile antibiotics. Trans. Br. Mycol. Soc. 57 : 41-48.

Dennis, C. et J. Webster. 1971c. Antagonistic properties of species groups of Trichoderma. III. Hyphal interactions. Trans. Br. Mycol. Soc. 57 : 363-369.

Gindrat, D. 1977. Effet de concentrations élevées de sels sur la croissance, la sporulation et la pigmentation de Trichoderma spp. Can. J. Microbiol. 23 : 607-616.

Gravel, V., C. Martinez, H. Antoun et R.J. Tweddell. 2005. Stimulation de la croissance de plants de tomate en hydroponie par le Pseudomonas putida et le Trichoderma atroviride. $97^{\mathrm{e}}$ Assemblée annuelle de la Société de protection des plantes du Québec, 9 et 10 juin 2005. Phytoprotection $86:$ 71-79.

Harman, G.E. 2006. Overview of mechanisms and uses of Trichoderma spp. Phytopathology 96 : 190-194.

Hibar, K., M. Daami-Remadi, H. Khiareddine et M. EI Mahjoub. 2005. Effet inhibiteur in vitro et in vivo du Trichoderma harzianum sur Fusarium oxysporum f. sp. radicis-lycopersici. Biotechnol. Agron. Soc. Environ. 9 (3) : 163-171.

Hmouni, A., M. Massoui et A. Douira. 1999. Étude de l'activité antagoniste de Trichoderma spp. et de Gliocladium spp. à l'égard de Botrytis cinerea, agent causal de la pourriture grise de la tomate. Al Awamia $100: 75-92$.

Kleifeled, O. et I. Chet. 1992. Trichodema harzianum Interaction with plants and effects on growth response. Plant Soil 144 (2) : 267-272.

Lamy Krafft, P. et M.F. Roquebert. 1981. Analyse des interactions entre deux champignons antagonistes Trichoderma viride Pers. et Botrytis cinerea Pers. Ex Fr. Études préliminaires. Cryptogam. Mycol. 2 : 137-151.

Lynch, J.M., R.D. Lumsden, P.T. Atkey et M.A. Ousley. 1991a. Prospects for control of Pythium damping-off of lettuce with Trichoderma, Gliocladium and Enterobacter spp. Biol. Fertil. Soils 12 : 95-99.

Lynch, J.M., K.L. Wilson, M.A. Ousley et J.M. Whipps. 1991b. Response of lettuce to Trichoderma treatment. Lett. Appl. Microbiol. 12 : 56-61.
MacKenzie, A.J., T.W. Starman et M.T. Windham. 1995. Enhanced root and shoot growth of Chrysanthemum cutting propagated with the fungus Trichoderma harzianum. Am. Soc. Hortic. Sci. 30 (3) : 496-498.

McGovern, R.J., L.E. Datnoff et L. Tripp. 1992. Effect of mixed infection and irrigation method on colonization of tomato roots by Trichoderma harzianum and Glomus intraradix. Proc. Fla. State Hortic. Soc. 105 : 361-363.

Messiaen, C.M. et R. Lafon. 1965. Les maladies des plantes maraîchères. INRA, Paris. Tome 2.

Mouria, A., A. Ouazzani-Touhami, A. Douira, R. Benkirane, A. Mlaiki et M. El Yachioui. 1997a. Antagonisme in vitro de Trichoderma spp. vis-à-vis de P. oryzae. Al Awamia 96 : 9-17.

Mouria, A., A. Ouazzani-Touhami, A. Mlaiki, M. El Yachioui et A. Douira. 1997b. Lutte biologique contre Helminthosporium oryzae : Antagonisme in vivo des Trichoderma spp. vis-à-vis de l'H. oryzae. Troisième congrès de I'Association Marocaine de Protection des Plantes, Rabat, 23-24 déc. P. 113-116.

Nemec, S., L. Datnoff et J. Strandberg. 1996. Efficacy of biocontrol agents in planting mixes to colonize plant roots and control root diseases of vegetables and citrus. Crop Prot. $15:$ 735-742.

Ouazzani-Touhami, A., A. Douira, R. Benkirane, M. El Oirdi, F. Bouslim, L. Zidane, N. Gmira et N.E. El Haloui. 1994. Antagonisme in vivo de certaines espèces fongiques visà-vis de Verticillium dahliae. Rev. Rés. APAMA 7 : 197211.

Ouazzani-Touhami, A., A. Mouria, A. Douira, R. Benkirane, A. Mlaiki et M. El Yachioui. 1997a. Influence du pH et de la température sur l'aptitude de Trichoderma spp. à inhiber in vitro la croissance mycélienne de $P$. oryzae. Al Awamia 96 : 19-24.

Ouazzani-Touhami, A., A. Mouria, A. Douira, A. Hmouni, A. Mlaiki et M. El Yachioui. 1997b. Antagonisme in vivo des Trichoderma vis-à-vis de P. oryzae. Al Awamia 96 : 25-31.

Ousley, M.A., J.M. Lynch et J.M Whipps. 1993. Effect of Trichoderma on plant growth; a balance between inhibition and growth promotion. Microb. Ecol. 26 : 277-285.

Ousley, M.A., J.M. Lynch et J.M. Whipps. 1994. Potential of Trichoderma spp. as consistent plant growth stimulators. Biol. Fertil. Soils 17 (1) : 85-90.

Ozbay, N. et S.E. Newman. 2004. The effect of the Trichoderma harzianum strains on the growth of tomato seedling. Acta Hortic. 635 : 131-135.

Paulitz, T., M. Windham et R. Baker. 1986. Effect of peat: vermiculite mixes containing Trichoderma harzianum on increased growth response of radish. J. Am. Soc. Hortic. Sci. 111 (5) : 810-816.

Windham, M.T., Y. Elad et R. Baker. 1986. A mechanism for increased plant growth induced by Trichoderma spp. Phytopathology 76 : 518-521.

Woo, S.L., A. Zoina, G. Del Sorbo, M. Lorito, B. Nanni, F. Scala et C. Noveiello. 1996. Characterization of Fusarium oxysporum f. sp. phaseoli by pathogenic races, VCGs, RFLPs, and RAPD. Phytopathology 86 : 966-972.

Yedidia, I., N. Benhamou et I. Chet. 1999. Induction of defence responses in cucumber plant (Cucumis sativus L.) by biocontrol agent Trichoderma harzianum. Appl. Environ. Microbiol. 65 : 1061-1070.

Zidane, L. 2004. Étude des groupements messicoles dominés par l'avoine stérile (Avena sterilis L. spp. macrocarpa Mo.) dans quelques régions du Maroc occidental et de la compétitivité de cette adventice vis-à-vis du blé dur. Thèse de Doctorat d'État Es-Sciences Naturelles. Université Ibn Tofaïl, Faculté des Sciences, Kénitra. 178 pp. 